\section{Integrative Medicine \\ International}

\title{
Rapid Treatment of Waist Pain by Nanoscale Electric Stimulation
}

\author{
Jaehwi Lee ${ }^{a} \quad J a e$ Youl Cho ${ }^{c}$ Kyoung Won Kim ${ }^{b}$ \\ ${ }^{a}$ College of Pharmacy, Chung-Ang University, and ${ }^{b}$ Research Institute, Human \\ Nanoelectrotech Co. Ltd., Seoul, and ' Department of Genetic Engineering, Sungkyunkwan \\ University, Suwon, Korea
}

Key Words

Waist pain · Electrical stimulation · Metallic ion band · Aluminum and copper bands

\section{Abstract}

The primary purpose of the study was to examine the ability of thin aluminum and copper bands to treat difficulties in moving, such as walking, standing and sitting, due to waist pain. The mechanism behind this therapy is a very weak nanoscale electrical stimulation caused by the electric current flow between aluminum and copper due to a difference in ionization tendency and electronegativity. The bands were firmly attached at the specified area, which was carefully selected based on the nerve connection of the human body. Prompt improvement in those having moving difficulties could be observed. More clinical trials are being conducted to analyze the rapid effect of nanoelectric current on the treatment of pain.

(c) 2014 S. Karger AG, Basel

\section{Introduction}

The human body consists of numerous cells that are tightly linked to one another, and therefore, the body electricity flows throughout the cell linkage at various current levels (15 $\mathrm{nA}$ to $2 \mu \mathrm{A}$ ). A well flowing body current is considered as one of the crucial elements to maintain a healthy body. Abnormal body current, such as no current or small and large amounts of body current, can cause problems at particular body sites resulting in pain [1]. 
Fig. 1. Nerve connection of the human body. The capital letters C, T, L, and S stand for the cervical, thoracic, lumbar, and sacrum nerves.

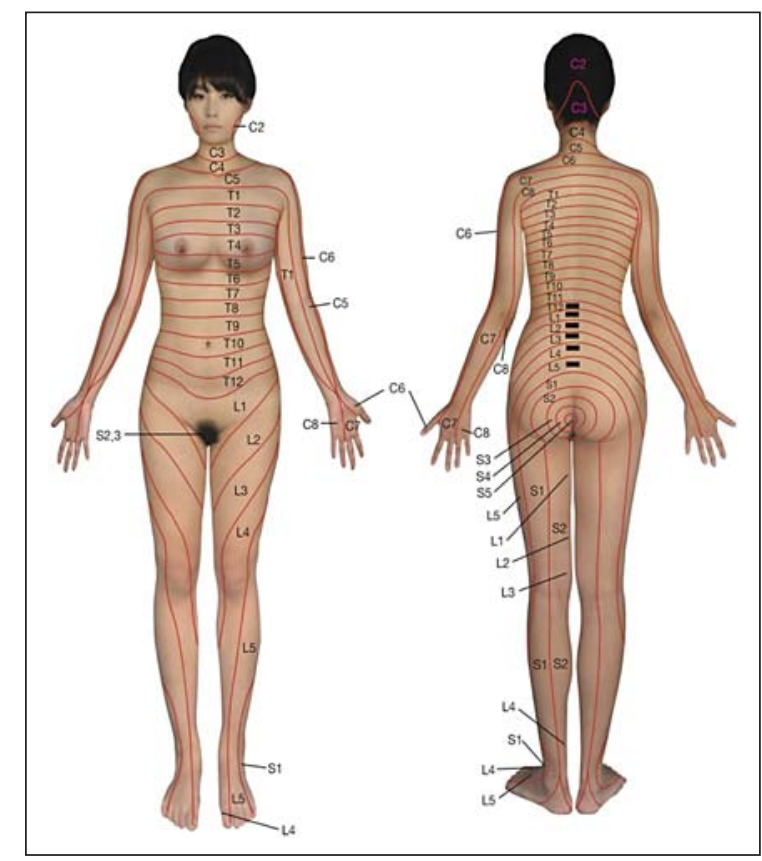

Becker and Selden [2] have published a book entitled 'The Body Electric: Electromagnetism and the Foundation of Life' in which they say that electric current in living things causes the healing process of the human body essential to longer and healthy life. This book has generally and frequently been referred to when attempting to understand the physical and psychiatric phenomena and healing process of our body.

We have demonstrated that electrical stimulation with a weak current induced by thin aluminum and copper patches is able to change signal transduction pathways related to inflammation responses; thus, electrical stimulation can be used for the treatment of a wide range of inflammation diseases [3]. Using different metal plates, it would be possible to induce a current similar to the normal levels. In a disease state, the body current does not flow well or is blocked sometimes, probably causing pain or paralysis. Allowing normal body current would provide pain relief [4].

Based on our experience and knowledge obtained from embryology and nerve anatomy, we recognized that the nerve system could be classified into four different connection groups (CGs) of which each group regulates different organs. We named the CGs as follows: CG 1, 2, 3 , and 4 . The body current flows through each group, and thus, each CG can also be called a current line.

CG 1 is a nerve connection between lumbar 4 (L4) and the thoracic 1 (T1) nerve section and controls the stomach, esophagus, liver, heart, and breast. Likewise, CG 2 is a nerve connection between sacrum 2 (S2) and cervical 6 (C6) and controls the lung, kidney, colon, uterus, vagina, and testis. CG 3 is a nerve connection between cervical 8 (C8) and sacrum 1 (S1) and regulates the prostate gland, duodenum, small intestine, spine, nose, and blood vessels. Finally, CG 4 is a nerve connection between cervical 7 (C7) and lumbar 5 (L5) and controls the bile duct, lymphatic system, arms and legs. Figure 1 shows the nerve connections in the human body.

Based on the background mentioned above, we have designed metallic ion bands with thin aluminum and copper that are able to generate nanoscale electric current flow and have used them for the treatment of waist pain, one of the main pain diseases in elderly individuals. 
Fig. 2. Application of rectangular aluminum bands to the waist muscles.

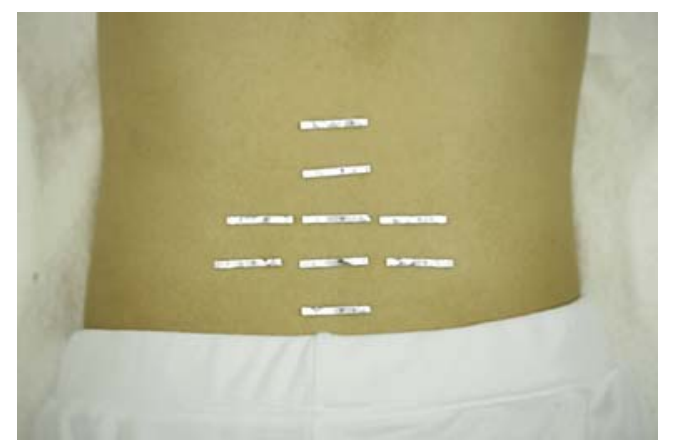

\section{Preparation of the Aluminum and Copper Bands}

Copper was selected because its electronegativity is larger than that of aluminum, the other metal band chosen in this study. A copper plate (with a purity of $99.96 \%$ and free from lead, cadmium, mercury, and hexavalent chromium) with a thickness of $0.5 \mathrm{~cm}$ was cold rolled to produce a thin copper film with a thickness of $0.1 \mathrm{~mm}$, and it was attached with an adhesive to one side of a soft fabric sheet.

An aluminum band was used as its ionization tendency is greater than that of copper. An aluminum plate (with a purity of $99.7 \%$ ) with a thickness of $0.5 \mathrm{~cm}$ was also cold rolled to form a thin aluminum film with a thickness of $0.1 \mathrm{~mm}$ and was attached to a soft adhesive fabric sheet. The aluminum band may concurrently contain a very limited amount of silicon, iron, copper, manganese, magnesium, zinc, and titanium.

The electric current flows from copper to aluminum due to a difference in the ionization tendency and electronegativity of copper and aluminum.

\section{Treatment with Aluminum and Copper Bands}

\section{Study Subjects}

Fifty subjects with severe to moderate pain evaluated by a questionnaire participated in this study. The severity of pain was determined with a 0-10 numeric rating scale set by the Warren Grant Magnuson Clinical Center, National Institutes of Health. A rating of 0 indicated no pain, while ratings of 1-3, 4-6, and 7-10 represented mild pain (annoying, interfering little with walking, standing, or sitting), moderate pain (interfering considerably with walking, standing, or sitting), and severe pain (disabling to walk, stand, or sit), respectively. Subjects' age ranged between 22 and 73 years. A total of 27 male and 23 female patients were enrolled after obtaining informed consent. The mean ages of the subjects were 48.5 years for the male and 47.2 years for the female patients. The body mass index values were 26 and 27 for males and females, respectively. Each patient had difficulties in walking, standing, or sitting due to waist pain.

\section{Application of the Aluminum and Copper Bands}

Nine aluminum bands ( $0.5 \mathrm{~cm}$ width, $5 \mathrm{~cm}$ length) were first attached to the waist muscle as shown in figure 2. Afterwards, round-shaped copper and aluminum patches $0.8 \mathrm{~cm}$ in diameter) were simultaneously attached to the fingers and toes of the left and right hands and feet as shown in figure 3 . 
Fig. 3. Application of roundshaped aluminum and copper bands to the fingers and toes. Numbers represent the attachment points of the nerve CGs with the same numbers.

Table 1. Changes in the degree of waist pain before and after the treatment with aluminum and copper bands

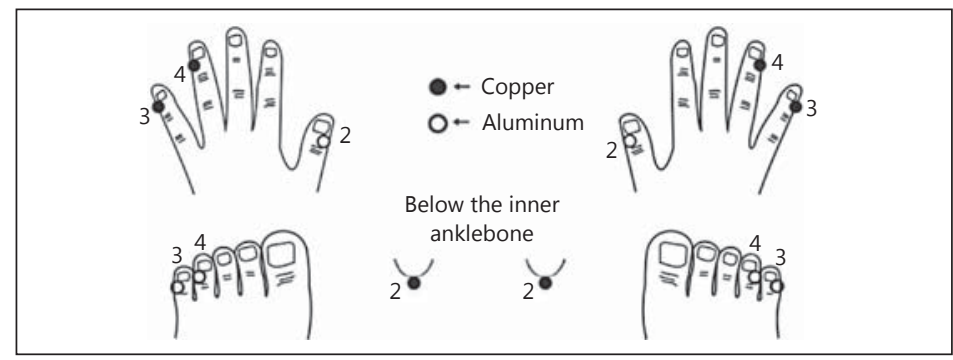

Degree of improvement of waist pain

\begin{tabular}{llrl} 
& & $(\mathrm{n}=13)$ & $(\mathrm{n}=16)$ \\
Excellent & 7 & 10 & 4 \\
Better & 2 & 5 & 1 \\
Good & 1 & 4 & 0 \\
Fair & 1 & 1 & 2 \\
No change & 2 & 1 & \\
\hline
\end{tabular}

The degree of the improvement of waist pain was evaluated following the attachment of aluminum and copper bands and patches (see fig. 2 and 3). The decision on the degree of improvement was made based on the responses using a 0-10 numeric rating scale before treatment. When the patient reported the same degree of pain before and after the treatment, it was regarded as no change.

After attaching the metal bands to the hands and feet, the differently colored bands were attached also. Consequently, when gold-colored copper patches are attached to the fingers of the hand, the silver-colored aluminum patches should be attached to the toes of the feet to generate a nano-level current and enable its flow throughout the body.

\section{Evaluation of Changes in the Degree of Pain}

Changes in the degree of waist pain were evaluated by a questionnaire and after observing the behaviors or performance of patients who received aluminum and copper band treatment as a function of time.

\section{Clinical Effect of Aluminum and Copper Bands on the Improvement of Waist Pain}

\section{Degree of the Improvement of Waist Pain}

In our previous study [3], we demonstrated that a nanoscale electric current of 35-55 nA could be induced by placing aluminum and copper patches in different media such as saline or cell culture media (RPMI-1640 supplemented with 10\% inactivated fetal bovine serum, glutamine, penicillin, and streptomycin). The induction of the nanoscale electric current was probably due to ionization of the metals. Some researchers have already shown the generation of ions from a wide range of metals and their activities [5, 6]. 
Table 2. Time required to recognize the improvement of waist pain in patients whose degrees of improvement were excellent, better, good, or fair

\begin{tabular}{llll}
\hline $\begin{array}{l}\text { Time required to } \\
\text { recognize the } \\
\text { improvement }\end{array}$ & $\begin{array}{l}\text { Walking } \\
(\mathrm{n}=11)\end{array}$ & $\begin{array}{l}\text { Standing } \\
(\mathrm{n}=20)\end{array}$ & $\begin{array}{l}\text { Sitting } \\
(\mathrm{n}=14)\end{array}$ \\
\hline$<10 \mathrm{~s}$ & 9 & 14 & 11 \\
$<30 \mathrm{~s}$ & 0 & 2 & 2 \\
$<1 \mathrm{~min}$ & 0 & 1 & 0 \\
$<10 \mathrm{~min}$ & 2 & 1 & 1 \\
$>10 \mathrm{~min}$ & 0 & 2 & 0 \\
\hline
\end{tabular}

The changes in the degree of the waist pain before and after the treatment with aluminum and copper bands and patches are presented in table 1.

As shown in table 1 , about 69,71 , and $81 \%$ of the patients having difficulties with, respectively, walking, standing, and sitting due to waist pain responded that their problems could be improved and reach the degrees of excellent and better after the attachment of the aluminum and copper bands and patches.

This improvement can probably be attributed to the induced electric currents that cause a normal electric current flow in the body. A similar amelioration has been confirmed by several other studies [7-9]. Therefore, it can be stated that the normalization of the body's electric flow possesses an ability to reduce chronic pain such as waist pain and inflammatory symptoms.

Approximately 4.7-15.4\% of the respondents experienced no changes in their symptoms. Reasons for ineffectiveness may stem from an individual difference in the location of nerve lines.

Since these results have been obtained in a limited number of patients, the degree of effectiveness should be further analyzed in a larger number of patients.

\section{Improvement Time}

Table 2 clearly demonstrates that the improvement of waist pain occurred very fast such that most (i.e., 80-93\%) of the patients recognized an amelioration of symptoms after less than $30 \mathrm{~s}$.

This result indicates that the process involved in the induction of electric current using aluminum and copper bands and the normalization of the flow of the body electric current occurred very rapidly.

\section{Conclusion}

We investigated the usefulness of the aluminum and copper bands as a platform to generate nanoscale electric current that can normalize the flow of the electric current in the body. Most of the patients having difficulties in walking, standing, and sitting due to waist pain rapidly experienced symptom improvement. Therefore, it can be concluded that the nanoscale electric induction is a promising treatment method for curing pain.

Further studies are being conducted to confirm the effectiveness obtained in the present study and to extend the current results to other pain diseases. 


\section{Acknowledgement}

This study was reviewed by Prof. Weidong Pan (Shanghai, China) and Prof. Majid Avijgan (Iran).

\section{Disclosure Statement}

The authors have no conflicts of interest to disclose.

\section{References}

1 Kim KW: Functional metallicity ion band. US Patent, Patent No. 07904173, 2011.

2 Becker RO, Selden G: The Body Electricity - Electromagnetism and the Foundation of Life. New York, William Morrow and Company, Inc., 1985.

3 Jeong D, Lee J, Yi Y-S, Yang Y, Kim KW, Cho JY: p38/AP-1 pathway in lipopolysaccharide-induced inflammatory responses is negatively modulated by electrical stimulation. Mediators Inflamm 2013;2013:183042.

4 Kouwenhoven WB: Effects of electricity on the human body. Electr Eng 1949;68:199-203.

5 Berger TJ, Spadaro JA, Bierman R: Antifungal properties of electrically generated metallic ions. Antimicrob Agents Chemother 1976;10:856-860.

-6 Berger TJ, Spadaro JA, Chapin SE, Becker RO: Electrically generated silver ions: quantitative effects on bacterial and mammalian cells. Antimicrob Agents Chemother 1976;9:357-358.

-7 Shafer N, Kitay G: Transcutaneous electrical nerve stimulation and pain relief: an overview. Med Electron 1988;19:132-136.

8 Khadilkar A, Milne S, Brosseau L: Transcutaneous electrical nerve stimulation (TENS) for chronic low-back pain. Cochrane Database Syst Rev 2005;3:CD003008.

-9 Sarzi-Puttini P, Cimmino MA, Scarpa R, et al: Osteoarthritis: an overview of the disease and its treatment strategies. Semin Arthritis Rheum 2005;35(1 Suppl 1):1-10. 